Zagazig J. Agric. Res., Vol. 43 No. (1) 2016

http:/www.journals.zu.edu.eg/journalDisplay.aspx? Journalld=1\&queryType=Master

\title{
EFFICIENCY OF THREE BIOINSECTICIDES SPRAY IN PREHARVEST TO CONTROL PEACH FRUIT FLY DURING POSTHARVEST OF GUAVA SEEDLESS FRUITS
}

\author{
Naglaa M.A. Yassin ${ }^{1 *}$ and Anas A. Ahmed ${ }^{2}$ \\ 1. Hort. Res. Station, Sabahia, Alex., Hort. Res. Inst., ARC. Giza, Egypt \\ 2. Plant Prot. Inst., Agric. Res. Cent., Station, Sabahia., ARC. Giza, Egypt
}

\begin{abstract}
This experiment was carried out during 2013 and 2014 seasons on guava seedless fruits to study the effect of spray of three bioinsecticides; Biozeid 2.5\%, Pritecto 9.4\% and Bioarc 6\% three levels each (300, 250 and 200g/100 l water) at preharvest to control peach fruit fly during postharvest of guava fruits. Stored fruits at $8^{\circ} \mathrm{C}$ and $\mathrm{RH} .85-90 \%$. Weight loss,firmness,total soluble solides, carotenoids, acidity and vitamin $\mathrm{C}$, were determind. The highest effect for controlling peach fruit fly observed with Bioarc 6\% treatments of guava fruits in both seasons. The results indicated that, there was lower weight loss with fruits which were treated by Biozeid 2.5\% and by Pritecto 9.4\%. Firmness, carotenoids and vitamin $\mathrm{C}$. were significantly higher with fruits treated by Bioarc $6 \%$, but total soluble solid was significantly higher in fruits treated by Biozeid $2.5 \%$ in both seasons. In both seasons, weight loss percentage, total soluble solid contents (TSS) and carotenoids were increased significantly by the advancing of storage periods but firmness, total acidity and vitamin C. decreased during storage.
\end{abstract}

Key words: Bioinsecticides, peach fruits fly, biozeid, pritecto, bioarc.

\section{INTRODUCTION}

Guava (Psidium guajava L.) is an important resource in the domestic economy of many countries in the tropics (Yavada, 1996). Guava is a very popular fruit, it is, generally a good source of lycopene, beta carotene, vitamin C, protein, fats, carbohydrate, fibers, minerals and vitamin B. In Egypt, guava trees are widely planted especially in Beheira, Elsharkia, around Alexandria and newly reclaimed lands. In Egypt guava occupy about 38000 faddans, yielded about 314000 tons as annual fruit production with an exported range about 16.312.38 metric tons to many countries (Cheaour et al., 1990). Guava exports from Egypt are increased through air flight as the main transport system. The limiting factor for export is the high costs that reduce the profit level to the grower. Refrigeration appears to be a suitable way for sea transport. The guava is highly perishable, susceptible to mechanical damage and chilling injury and has a limited postharvest shelf life. The peach fruit fly Bacterocero zomata (saunders) in damagerous pest for many fruit crops in different countries. Control depends upon bioinsecticides (Mahmoud, 2009). The insect feeds on the internal fruit contents. $\mathrm{Al} \mathrm{l}$ damaged fruit must be destroyed to break the cycle of infection. Remove any fruit from the tree with dimples or weeping clear sap as this is a sign that egges have already been laid in the fruit (Rahmann and Akhsoy, 2014). The aim of this investigation was to study the effects of bio insecticied spray on preharvest and postharvest guava fruits to control the peach fruit flies.

\footnotetext{
Corresponding author: Tel. : +201097210752

E-mail address: nagla_ys@yahoo.com
} 


\section{MATERIALS AND METHODS}

The investigation was carried out during 2013 and 2014 seasons on seedless guava fruits (Psidium guajava L.). Fruits were harvested from plants grown in Sabahia station, Alexandria. The experiment was conducted in four feddans contained of guava. It harvested at mature stage, according to Robert and Ching (2014). Fruits were immediately transported to the laboratory of handling and postharvest at sabahia station Alexandria, horticulture research of Giza, Egypt. Fruits were sorted for size uniformity and absence defects. Sound selected fruits were washed using fresh tap water, and air dried. Then fruits divided into four groups each group contained six fruits. Guava fruits were received the following treatments at preharvest and postharvest as follows:

At preharvest, three bioinscticides were used Biozeid 2.5\%, Pritecto 9.4\% and Bioarc 6\% and sprayed with three concentrations 300, 250 and $200 \mathrm{~g} / 100 \mathrm{l}$ water, each separately.

A week beforharvest, fruits were spraied with bioinscticides, then all treatments stored at $8^{\circ} \mathrm{C}$ and RH. (85-90\%).

Each treatments were packed in three carton boxes at dimension of $(50 \times 30 \times 20 \mathrm{~cm})$ each box contain six fruits represented one replicate and all treatments stored at $8^{\circ} \mathrm{C}$. The changes in physical and chemical properties of fruits were followed up each seven days interval through the experimental period as following:

\section{Physical Characteristics}

Weight loss percentage (\%): It was calculated according to the following equation:

$$
\text { Weight loss (\%) }=\frac{\text { Initial weight- weight at sampling date }}{\text { Initial weight }} \times 100
$$

Three fruits were labeled in every replicate and initially weighted to calculate the fruits weight loss percentage during the cold storage in relation to its original weight.

\section{Firmness $\left(\mathrm{g} / \mathrm{cm}^{2}\right)$}

Pulp texture: was determined by Lifra texture analyzer instrument using a penetrating cylinder of $1 \mathrm{~mm}$ in diameter, to a constant distance (2 $\mathrm{mm}$ ) inside the pulp of fruits, and by a constant speed $2 \mathrm{~mm}$ per sec., and the peak of resistance was recorded per g.

\section{Determining the Infestation of Fruits by Peach Flies}

Is known by the number of holes in the fruit itself were recorded the more number of holes means the more infestation percentage and the number of eggs increase.

\section{Chemical Properties}

\section{Total soluble solids content (TSS)}

The obtained juice was used to determine the percentage of Total soluble solids content (TSS) by the use of a hand refractometer according to Chen and Mellenthin (1981)

\section{Titratable acidity (\%)}

Total acidity was determined by titrating 5 $\mathrm{ml}$ of the extracted juice against $0.1 \mathrm{~N}$ of $\mathrm{NaOH}$ using phenol phethalin indicator, titratable acidity was expressed as percentage of citric acid (g citric acid/100 ml juice) according to (AOAC, 2005).

\section{Ascorbic acid content}

Five $\mathrm{ml}$ of sample of fruit juice $+5 \mathrm{ml}$ metaphosphric acid were added to each sample, which was titrated with 2.6 dichlorophenol indophenol solution. Ascorbic acid content was expressed as milligrams of ascorbic acid per 100 $\mathrm{ml}$ of fruits juice, according to (AOAC, 2005).

\section{Carotenoids content}

Carotenoids content of fruits pulp was extracted by direct dipping of $10 \mathrm{~g}$ of blended fruit pulp into solution containing $(40 \mathrm{ml}$ acetone, $60 \mathrm{ml}$ hexane and $0.1 \mathrm{~g} \mathrm{Mg} \mathrm{CO}_{3}$ and blended for $5 \mathrm{~min}$. to determined by colorimeter according to (AOAC, 2005).

\section{Decay percentage}

Determined by count the number of fruits decayed and expressed as percentage from sound fruits.

\section{Statistical Analysis}

The experimental design was factorial Randomized complete bloch design (RCBD) with three replicates, and all the obtained data were statistically analyzed according to Snedecor and Cochran (1980). The individual 
comparisons were carried out by using the least significant difference (LSD) according to SAS Institute (1985).

The experiment was distributed in split -split design and the means were compared by LSD at 0.05 according to Steel and Torrie (1980).

\section{RESULTS}

\section{Mean of Three Replicates + SE}

The data was recorded in Table 1 reveal that There were highly significant differences between the three bio-insecticides and the means can be arranged according to their effects as follows at first season 23.11 flies for Biozeid 2.5\%, 22.55 flies for Pritecto 9.4\% and (17.66) for Bioarc $6 \%$. The second season was highly significant 43.56 for Biozeid 2.5\%, 7.56 for Bio arc $6 \%$ and 5.56 for Pritecto $9.4 \%$ this result are in line with Hanafy et al. (2009).

\section{Grand Mean of Replicates + SE}

The result shown in Table 2 clear that low grand means of peach fruit fly at first season (33.92 flies) and (32.44 flies) for second season at first concentration; at second concentration first season recorded 39.50 flies while 39.17 flies were recorded for second season and the third concentration in first season were 44.41 flies and 42.75 flies for second season. These results agreed with those reported by Hanafy et al. (2009).

\section{Physical Characteristics}

\section{Fruit weight loss percentage}

Data presented in Table 3 show that, the two seasons, weight loss percentage had lower in fruits were treated (Biozeid 2.5\%, Pritecto 9.4\% and Bioarc 6\%) with concentration 200g/100 l water compared with untreated fruits in both seasons these results were agree with Khyber (2014).

The weight loss percentage was gradually increased as an average for all treatments, by increasing the storage period. The differences among all storage periods were significant in both seasons of study. The weight loss attributed respiration and the higher storage temperature. The later result agree with those reported by ElYaten and Kader (1984), El-Saedy and ElNaggar (2005).

\section{Fruit firmness}

From the tabulated data in Table 4, it was noticed that fruits treated with Bioarc 6\% (300g/ l water) had the highest value of firmness compared with all treatments in both seasons. This result agree with Singh and Pal (2009). Fruit firmness of all treated fruits was decreased with the duration in this really cold storage in both seasons. Similar, results were found by Rippon and Trochoulias, (2007) and Yassin and Tayel (2011). As a conclusion, loss in fruits firmness with the decomposition progress of storage period is mainly due to, enzymatic degredation of insoluble protopectins to more simple soluble pectin, solubilization of cell and cell wall contents as a result of the increasing in pectin esterase activity and subsequent development of juiciness and loss in peel and pulp hardness. These results were in agreement with those previously found by many researches such as Ponomarev (1968) and Siddiqui et al. (1996).

\section{Chemical Characteristics}

\section{Total soluble solids contents (TSS)}

The data illustrated in Table 5 show that soluble solids contents TSS in (untreated) fruits control and fruits were treated Bioarc 6\% were significantly higher compared with all treatments in both seasons. Esam Eldin and Osman (2014). TSS in the fruits by the storage periods was more than three folds its initial value at beginning of the storage period. The above finidings are in harmony with those of Lopez et al. (2003) on cactus pear and David and Whyte (2009).

\section{Carotenoids content}

The changes in carotenoids content of guava fruits in the two seasons of the study are presented in Table 6. Generally, there were higher significant differences between fruits were treated with Biozeid 2.5\%, Pritecto 9.4\%, and Bioarc $6 \%$ compared with untreated fruits.

These results agreed with Pen et al. (1999). It was noticed that carotenoids content were significantly increased during storage periods in both seasons. Similar results were found by Nurul and Mosharraf (2012) on banana. 
Table 1. Efficiency of certain biological insecticides spray on guava trees against Peach fruit fly Bacterocera zonata at two seasons (2013 and 2014)

\begin{tabular}{|c|c|c|c|c|}
\hline \multirow[t]{2}{*}{ Bio-insecticide } & \multirow{2}{*}{$\begin{array}{l}\text { Concentrations } \\
\text { g/100 I water }\end{array}$} & \multicolumn{2}{|c|}{ The mean number of B.Z. flies in traps } & \multirow[t]{2}{*}{ Grand mean } \\
\hline & & First season (2013) & Second season (2014) & \\
\hline \multirow{4}{*}{ Biozeid 2.5\% } & 300 & 15.67 & 38.00 & 26.84 \\
\hline & 250 & 21.67 & 44.33 & 33.00 \\
\hline & 200 & 32.00 & 48.33 & 40.17 \\
\hline & Mean & 23.11 & 43.56 & $33.34 b$ \\
\hline \multirow{4}{*}{ Pritecto 9.4\% } & 300 & 16.33 & 1.67 & 9.00 \\
\hline & 250 & 23.00 & 6.33 & 14.67 \\
\hline & 200 & 28.33 & 8.67 & 18.50 \\
\hline & Mean & 22.55 & 5.56 & $14.06 \mathrm{c}$ \\
\hline \multirow{4}{*}{ Bioarc 6\% } & 300 & 11.33 & 4.00 & 7.67 \\
\hline & 250 & 19.33 & 6.00 & 12.67 \\
\hline & 200 & 22.33 & 12.67 & 17.50 \\
\hline & Mean & 17.66 & 7.56 & $12.61 d$ \\
\hline \multirow{4}{*}{ Control } & & 92.33 & 98.00 & 95.17 \\
\hline & & 94.00 & 99.67 & 96.84 \\
\hline & & 95.67 & 101.33 & 98.50 \\
\hline & Mean & 94.00 & 99.67 & $96.84 a$ \\
\hline Grand mean & & 39.33a & $39.09 b$ & \\
\hline
\end{tabular}

LSD 0.05 for seasons $=0.12$, LSD 0.05 for bioinsecticide $=1.00$

Table 2. Evaluation of three bioinsecticides against Peach fruit fly Bacterocera zonata at two seasons in the field

\begin{tabular}{llcccc}
\hline Season & Bioinsecticide & \multicolumn{3}{c}{ Concentration } & Grand mean \\
\cline { 3 - 5 } & & $\mathbf{3 0 0} \mathbf{g}$ & $\mathbf{2 5 0} \mathbf{g}$ & $\mathbf{2 0 0} \mathbf{g}$ & \\
\hline & Biozeid 2.5\% & 15.67 & 21.67 & 32.00 & 23.11 \\
& Pritecto 9.4\% & 16.33 & 23.00 & 28.33 & 22.56 \\
First season (2013) & Bioarc 6\% & 11.33 & 19.33 & 22.33 & 17.67 \\
& Control & 92.33 & 94.00 & 95.00 & 94.00 \\
& Mean & 33.92 & 39.50 & 44.41 & $39.28 \mathrm{a}$ \\
& Biozeid 2.5\% & 38.00 & 44.33 & 48.33 & 43.56 \\
& Pritecto 9.4\% & 1.67 & 6.33 & 8.67 & 5.56 \\
Second season (2014) & Bioarc 6\% & 4.00 & 6.33 & 12.67 & 7.67 \\
& Control & 98.00 & 99.67 & 101.33 & 99.67 \\
& Mean & 32.44 & 39.17 & 42.75 & $39.11 \mathrm{~b}$ \\
Grand mean & & $34.67 \mathrm{c}$ & $39.34 \mathrm{~b}$ & $43.58 \mathrm{a}$ & \\
\hline
\end{tabular}

LSD 0.05 for concentration $=0.92$, LSD 0.05 for seasons $=0.12$ 
Table 3. Effect of three bioinsecticides spray in preharvest to control peach fruit fly during postharvest on weight loss (\%) of guava fruits during 2013 and 2014 season

\begin{tabular}{|c|c|c|c|c|c|}
\hline \multirow[t]{2}{*}{ Treatment g/100 I water } & \multicolumn{5}{|c|}{ Storage period (week) } \\
\hline & 1 & 2 & 3 & 4 & Means (B) \\
\hline & \multicolumn{5}{|c|}{ Season 2013} \\
\hline Biozeid 2.5\% 300 & 0 & 3.9 & 4.77 & 8.10 & $4.19 \mathrm{a}$ \\
\hline Biozeid 2.5\% 250 & 0 & 3.5 & 4.60 & 8.0 & $4.03 \mathrm{~b}$ \\
\hline Biozeid 2.5\% 200 & 0 & 3.0 & 4.5 & 7.8 & $3.83 c$ \\
\hline Pritecto 9.4\% 300 & 0 & 4.10 & 4.83 & 7.50 & $4.18 \mathrm{a}$ \\
\hline Pritecto $9.4 \% 250$ & 0 & 4.00 & 4.50 & 7.00 & $3.88 \mathrm{a}$ \\
\hline Pritecto 9.4\% 200 & 0 & 3.90 & 4.30 & 6.80 & $3.75 b$ \\
\hline Bioarc 6\% 300 & 0 & 4.50 & 5.43 & 8.61 & $4.64 \mathrm{a}$ \\
\hline Bioarc 6\% 250 & 0 & 4.00 & 5.00 & 8.30 & $4.33 a$ \\
\hline Bioarc 6\% 200 & 0 & 3.80 & 4.80 & 7.80 & $4.10 \mathrm{~b}$ \\
\hline Control & 0 & 5.56 & 6.93 & 11.98 & $6.11 \mathrm{a}$ \\
\hline \multirow[t]{2}{*}{ Means (A) } & 0a & $3.76 b$ & $4.97 c$ & 8.19d & \\
\hline & \multicolumn{5}{|c|}{ Season 2014} \\
\hline Biozeid 2.5\% 300 & 0 & 3.50 & 3.79 & 6.84 & 3.53a \\
\hline Biozeid 2.5\% 250 & 0 & 3.20 & 3.10 & 6.20 & $3.13 b$ \\
\hline Biozeid 2.5\% 200 & 0 & 3.00 & 2.90 & 6.00 & $3.00 \mathrm{c}$ \\
\hline Pritecto 9.4\% 300 & 0 & 3.73 & 4.28 & 6.70 & $3.68 \mathrm{a}$ \\
\hline Pritecto 9.4\% 250 & 0 & 3.20 & 4.00 & 6.20 & $3.35 a$ \\
\hline Pritecto 9.4\% 200 & 0 & 3.00 & 3.70 & 5.80 & $3.13 b$ \\
\hline Bioarc 6\% 300 & 0 & 3.73 & 4.12 & 8.98 & $4.21 \mathrm{a}$ \\
\hline Bioarc 6\% 250 & 0 & 3.50 & 4.00 & 8.50 & $4.00 \mathrm{~b}$ \\
\hline Bioarc 6\% 200 & 0 & 3.30 & 3.50 & 8.30 & $3.78 \mathrm{c}$ \\
\hline Control & 0 & 5.20 & 5.00 & 10.90 & $5.28 \mathrm{a}$ \\
\hline Means (A) & 0a & $3.53 b$ & $4.19 c$ & $7.44 d$ & \\
\hline
\end{tabular}


Table 4. Effect of three bioinsecticides spray in preharvest to control peach fruit fly during postharvest on firmness of guava fruits during 2013 and 2014 season

\begin{tabular}{|c|c|c|c|c|c|}
\hline \multirow[t]{2}{*}{ Treatment g/100 I water } & \multicolumn{5}{|c|}{ Storage period (week) } \\
\hline & 1 & 2 & 3 & 4 & Means (B) \\
\hline & \multicolumn{5}{|c|}{ Season 2013} \\
\hline Biozeid 2.5\% 300 & 2.50 & 1.50 & 1.45 & 1.00 & $1.61 \mathrm{a}$ \\
\hline Biozeid 2.5\% 250 & 2.50 & 1.20 & 1.20 & 0.95 & $1.46 \mathrm{~b}$ \\
\hline Biozeid 2.5\% 200 & 2.50 & 1.10 & 1.10 & 0.93 & $1.40 \mathrm{c}$ \\
\hline Pritecto $9.4 \% 300$ & 2.50 & 1.27 & 1.85 & 1.30 & $1.73 a$ \\
\hline Pritecto $9.4 \% 250$ & 2.50 & 1.10 & 1.50 & 1.10 & $1.55 \mathrm{a}$ \\
\hline Pritecto 9.4\% 200 & 2.50 & 0.98 & 1.30 & 0.95 & $1.43 \mathrm{~b}$ \\
\hline Bioarc 6\% 300 & 2.50 & 2.33 & 1.73 & 1.50 & $2.02 \mathrm{a}$ \\
\hline Bioarc 6\% 250 & 2.50 & 2.10 & 1.30 & 1.20 & $1.78 \mathrm{a}$ \\
\hline Bioarc 6\% 200 & 2.50 & 0.98 & 1.20 & 1.10 & $1.45 a$ \\
\hline Control & 2.50 & 2.05 & 2.03 & 1.20 & $1.95 a$ \\
\hline \multirow[t]{2}{*}{ Means (A) } & 2.50 & $1.46 \mathrm{~b}$ & $1.56 \mathrm{c}$ & $1.12 \mathrm{~d}$ & \\
\hline & \multicolumn{5}{|c|}{ Season 2014} \\
\hline Biozeid 2.5\% 300 & 1.80 & 1.23 & 1.20 & 1.00 & $1.31 \mathrm{a}$ \\
\hline Biozeid 2.5\% 250 & 1.80 & 1.00 & 0.98 & 0.97 & $1.19 \mathrm{~b}$ \\
\hline Biozeid 2.5\% 200 & 1.80 & 0.95 & 0.93 & 0.95 & $1.16 \mathrm{~b}$ \\
\hline Pritecto 9.4\% 300 & 1.80 & 1.20 & 1.30 & 1.05 & $1.34 \mathrm{a}$ \\
\hline Pritecto 9.4\% 250 & 1.80 & 1.10 & 0.98 & 0.97 & $1.21 \mathrm{a}$ \\
\hline Pritecto 9.4\% 200 & 1.80 & 0.98 & 0.95 & 0.93 & $1.17 \mathrm{~b}$ \\
\hline Bioarc 6\% 300 & 1.80 & 1.78 & 1.72 & 1.30 & $1.65 a$ \\
\hline Bioarc 6\% 250 & 1.80 & 1.30 & 1.40 & 1.00 & $1.38 b$ \\
\hline Bioarc 6\% 200 & 1.80 & 1.20 & 1.20 & 0.98 & $1.29 b$ \\
\hline Control & 1.80 & 1.28 & 1.70 & 1.00 & $1.45 \mathrm{a}$ \\
\hline Means(A) & $1.80 \mathrm{a}$ & $1.20 \mathrm{~b}$ & $1.21 \mathrm{c}$ & $1.02 \mathrm{~d}$ & \\
\hline
\end{tabular}


Table 5. Effect of three bioinsecticides spray in pre harvest to control peach fruit fly during postharvest on total soluble solids (\%) of guava fruits during 2013 and 2014 season

\begin{tabular}{|c|c|c|c|c|c|}
\hline \multirow[t]{2}{*}{ Treatment g/100 l water } & \multicolumn{5}{|c|}{ Storage period (week) } \\
\hline & 1 & 2 & 3 & 4 & Means (B) \\
\hline & \multicolumn{5}{|c|}{ Season 2013} \\
\hline Biozeid 2.5\% 300 & 8.50 & 13.33 & 13.60 & 14.53 & $12.49 a$ \\
\hline Biozeid 2.5\% 250 & 8.50 & 13.20 & 13.20 & 14.30 & $12.3 \mathrm{~b}$ \\
\hline Biozeid 2.5\% 200 & 8.50 & 13.00 & 13.10 & 14.10 & 12.18 \\
\hline Pritecto 9.4\% 300 & 8.50 & 9.37 & 12.67 & 15.96 & $11.63 a$ \\
\hline Pritecto 9.4\% 250 & 8.50 & 9.10 & 12.40 & 15.50 & $11.38 \mathrm{a}$ \\
\hline Pritecto 9.4\% 200 & 8.50 & 9.00 & 12.20 & 15.20 & $11.22 b$ \\
\hline Bioarc 6\% 300 & 8.50 & 12.00 & 13.80 & 15.70 & $12.50 \mathrm{a}$ \\
\hline Bioarc 6\% 250 & 8.50 & 11.98 & 13.50 & 15.50 & $12.37 b$ \\
\hline Bioarc 6\% 200 & 8.50 & 11.50 & 13.30 & 15.30 & $12.15 b$ \\
\hline Control & 8.50 & 14.13 & 15.97 & 16.73 & 13.83a \\
\hline \multirow[t]{2}{*}{ Means(A) } & $8.50 \mathrm{a}$ & $11.66 b$ & $13.37 \mathrm{c}$ & $15.28 \mathrm{~d}$ & \\
\hline & \multicolumn{5}{|c|}{ Season 2014} \\
\hline Biozeid 2.5\% 300 & 14.20 & 15.26 & 15.97 & 16.43 & $15.46 \mathrm{a}$ \\
\hline Biozeid 2.5\% 250 & 14.20 & 14.90 & 15.50 & 16.20 & $15.20 \mathrm{a}$ \\
\hline Biozeid 2.5\% 200 & 14.20 & 14.80 & 15.30 & 16.10 & $15.10 \mathrm{~b}$ \\
\hline Pritecto $9.4 \% 300$ & 14.20 & 10.33 & 15.07 & 16.03 & $13.91 \mathrm{a}$ \\
\hline Pritecto $9.4 \% 250$ & 14.20 & 10.10 & 15.02 & 16.00 & $13.83 b$ \\
\hline Pritecto 9.4\% 200 & 14.20 & 10.00 & 14.98 & 15.98 & $13.79 c$ \\
\hline Bioarc 6\% 300 & 14.20 & 12.90 & 13.87 & 15.33 & $14.08 \mathrm{a}$ \\
\hline Bioarc 6\% 250 & 14.20 & 12.50 & 13.50 & 15.10 & $13.83 b$ \\
\hline Bioarc 6\% 200 & 14.20 & 12.20 & 13.20 & 15.00 & $13.65 b$ \\
\hline Control & 14.20 & 14.73 & 16.20 & 16.80 & $15.48 \mathrm{a}$ \\
\hline Means (A) & $14.20 \mathrm{a}$ & $13.77 \mathrm{~b}$ & $15.89 \mathrm{c}$ & $15.89 \mathrm{c}$ & \\
\hline
\end{tabular}


Table 6. Effect of three bioinsecticides spray in preharvest to control peach fruit fly during postharvest on carotenoids 100mg/ml of guava fruits during 2013 and 2014 season

\begin{tabular}{lccccc}
\hline Treatment g/100 l water & \multicolumn{5}{c}{ Storage period (week) } \\
\cline { 2 - 6 } & $\mathbf{1}$ & $\mathbf{2}$ & $\mathbf{3}$ & $\mathbf{4}$ & Means (B) \\
\cline { 2 - 6 } & & & Season 2013 & \\
Biozeid 2.5\% 300 & 2.50 & 3.58 & 4.22 & 4.71 & $3.75 \mathrm{a}$ \\
Biozeid 2.5\% 250 & 2.50 & 3.20 & 4.00 & 4.50 & $3.55 \mathrm{~b}$ \\
Biozeid 2.5\% 200 & 2.50 & 3.00 & 3.98 & 4.40 & $3.47 \mathrm{c}$ \\
Pritecto 9.4\% 300 & 2.50 & 3.48 & 4.18 & 4.95 & $3.78 \mathrm{a}$ \\
Pritecto 9.4\% 250 & 2.50 & 3.20 & 4.00 & 4.50 & $3.55 \mathrm{~b}$ \\
Pritecto 9.4\% 200 & 2.50 & 3.00 & 4.40 & 4.40 & $3.47 \mathrm{c}$ \\
Bioarc 6\% 300 & 2.50 & 3.84 & 4.89 & 4.89 & $3.91 \mathrm{a}$ \\
Bioarc 6\% 250 & 2.50 & 3.50 & 4.50 & 4.50 & $3.68 \mathrm{~b}$ \\
Bioarc 6\% 200 & 2.50 & 3.20 & 4.30 & 4.30 & $3.00 \mathrm{c}$ \\
Control & 2.50 & 2.71 & 3.87 & 4.40 & $3.37 \mathrm{a}$ \\
Means(A) & $2.50 \mathrm{a}$ & $3.27 \mathrm{~b}$ & $4.08 \mathrm{c}$ & $4.56 \mathrm{~d}$ &
\end{tabular}

Season 2014

\begin{tabular}{lccccc} 
Biozeid 2.5\% 300 & 2.90 & 3.37 & 4.47 & 4.38 & $3.78 \mathrm{a}$ \\
Biozeid 2.5\% 250 & 2.90 & 3.20 & 4.30 & 4.00 & $3.70 \mathrm{a}$ \\
Biozeid 2.5\% 200 & 2.90 & 3.10 & 4.20 & 3.98 & $3.55 \mathrm{~b}$ \\
Pritecto 9.4\% 300 & 2.90 & 3.66 & 4.52 & 4.70 & $3.95 \mathrm{a}$ \\
Pritecto 9.4\% 250 & 2.90 & 3.50 & 4.30 & 4.50 & $3.80 \mathrm{a}$ \\
Pritecto 9.4\% 200 & 2.90 & 3.20 & 4.00 & 4.20 & $3.58 \mathrm{~b}$ \\
Bioarc 6\% 300 & 2.90 & 3.32 & 4.12 & 4.90 & $3.81 \mathrm{a}$ \\
Bioarc 6\% 250 & 2.90 & 3.00 & 4.00 & 4.50 & $3.60 \mathrm{~b}$ \\
Bioarc 6\% 200 & 2.90 & 2.99 & 3.98 & 4.20 & $3.52 \mathrm{c}$ \\
Control & 2.90 & 2.72 & 3.79 & 4.37 & $3.45 \mathrm{c}$ \\
Means (A) & $2.90 \mathrm{a}$ & $3.24 \mathrm{~b}$ & $4.17 \mathrm{c}$ & $4.37 \mathrm{~d}$ & \\
\hline LSD 0.05 2013 A=0.82,B= 0.35 & LSD 0.05 2014 A=0.38,B=0.27 & & \\
\hline
\end{tabular}




\section{Titratable acidity (TA)}

The data presented in Table 7 indicated that titratable acidity percentages in treated guava in both seasons were significantly higher than untreated fruits. The obtained results are in agreement with Rosario et al. (2013). Reported that TA were higher than treatments on guava. The data pointed out also that there was slight decreased in TA percentage with the increase of storage period. Data could be attributed due to the increase of its consumption in respiration activities as an organic substrate. This result agreed with those reported by El-Seidy (2000) on peach, Nurul and Mosharraf (2012) on banana and Muhammad et al. (2012) on peach.

\section{Vitamin C}

The obtained data in Table 8 showed that ascorbic acid content value of guava, which treated with (Biozeid 2.5\%, Pritecto 9.4\% and Bioarc 6\%) and concentration 300g/100l water were higher significantly than untreated fruits.
The above mentioned results agree with those Rahmann and Akhsoy (2014). On the other hand, ascorbic acid content was decreased from the beginning and the end of experiment. These results are in good line with those observed by Nurul and Mosharraf (2012) on banana fruits. The reduction in vitamin c contents during ripening might be attributed to the oxidation of ascorbic acid as ripening proceeded in the untreated fruits.

\section{Decay of fruits}

Data pointed out in both seasons Table 9 clear, in both seasons, that untreated fruits had higher significantly values compared with all treatments and Preticto 9.4\% treatment had higher value of decay compared with Biozeid $2.5 \%$ and Bioarc 6\% this results agree with Michael and Palmateer (2 013). Advanced with storage period, all treatments increased, significantly. Omayma et al. (2010) came to similar results.

Table 7. Effect of three bioinsecticides spray in preharvest to control peach fruit fly during postharvest on titratable acidity (\%) of guava fruits during 2013 and 2014 season

\begin{tabular}{lccccc}
\hline Treatment g/100 l water & \multicolumn{5}{c}{ Storage period (week) } \\
\cline { 2 - 6 } & $\mathbf{1}$ & $\mathbf{2}$ & $\mathbf{3}$ & $\mathbf{4}$ & Means (B) \\
\hline & 0.72 & 0.42 & 0.38 & 0.30 & $0.46 \mathrm{a}$ \\
Biozeid 2.5\% 300 & 0.72 & 0.41 & 0.37 & 0.28 & $0.45 \mathrm{~b}$ \\
Biozeid 2.5\% 250 & 0.72 & 0.40 & 0.36 & 0.27 & $0.44 \mathrm{c}$ \\
Biozeid 2.5\% 200 & 0.72 & 0.40 & 0.32 & 0.26 & $0.43 \mathrm{a}$ \\
Pritecto 9.4\% 300 & 0.72 & 0.39 & 0.30 & 0.25 & $0.42 \mathrm{~b}$ \\
Pritecto 9.4\% 250 & 0.72 & 0.38 & 0.29 & 0.22 & $0.40 \mathrm{c}$ \\
Pritecto 9.4\% 200 & 0.72 & 0.37 & 0.27 & 0.24 & $0.40 \mathrm{a}$ \\
Bioarc 6\% 300 & 0.72 & 0.35 & 0.26 & 0.22 & $0.39 \mathrm{~b}$ \\
Bioarc 6\% 250 & 0.72 & 0.32 & 0.24 & 0.20 & $0.37 \mathrm{c}$ \\
Bioarc 6\% 200 & 0.72 & 0.70 & 0.54 & 0.46 & $0.61 \mathrm{a}$ \\
Control & $0.72 \mathrm{a}$ & $0.41 \mathrm{~b}$ & $0.48 \mathrm{c}$ & $0.27 \mathrm{~d}$ & \\
Means(A) & & & Season 2014 & & \\
& 0.60 & 0.37 & 0.35 & 0.30 & $0.41 \mathrm{a}$ \\
Biozeid 2.5\%300 & 0.60 & 0.35 & 0.31 & 0.29 & $0.39 \mathrm{~b}$ \\
Biozeid 2.5\% 250 & 0.60 & 0.32 & 0.30 & 0.27 & $0.37 \mathrm{c}$ \\
Biozeid 2.5\% 200 & 0.60 & 0.42 & 0.36 & 0.32 & $0.43 \mathrm{a}$ \\
Pritecto 9.4\% 300 & 0.60 & 0.40 & 0.35 & 0.30 & $0.41 \mathrm{~b}$ \\
Pritecto 9.4\% 250 & 0.60 & 0.38 & 0.32 & 0.27 & $0.39 \mathrm{c}$ \\
Pritecto 9.4\% 200 & 0.60 & 0.37 & 0.30 & 0.25 & $0.38 \mathrm{a}$ \\
Bioarc 6\% 300 & 0.60 & 0.31 & 0.29 & 0.22 & $0.36 \mathrm{~b}$ \\
Bioarc 6\% 250 & 0.60 & 0.30 & 0.25 & 0.20 & $0.34 \mathrm{c}$ \\
Bioarc 6\% 200 & 0.60 & 0.56 & 0.42 & 0.40 & $0.50 \mathrm{a}$ \\
Control & $0.60 \mathrm{a}$ & $0.38 \mathrm{~b}$ & $0.33 \mathrm{c}$ & $0.28 \mathrm{~d}$ & \\
Means (A) & & & & \\
\hline
\end{tabular}

LSD $0.052013 \mathrm{~A}=0.35, \mathrm{~B}=0.03$ LSD $0.052014 \mathrm{~A}=0.25, \mathrm{~B}=0.04$ 
Table 8. Effect of three bioinsecticides spray in preharvest to control peach fruit fly during postharvest on vitamin $C$ of guava fruits during 2013 and 2014 season

\begin{tabular}{|c|c|c|c|c|c|}
\hline \multirow[t]{2}{*}{ Treatment g/100 I water } & \multicolumn{5}{|c|}{ Storage period (week) } \\
\hline & 1 & 2 & 3 & 4 & Means (B) \\
\hline & \multicolumn{5}{|c|}{ Season 2013} \\
\hline Biozeid 2.5\% 300 & 72.00 & 60.50 & 57.00 & 54.67 & $61.17 a$ \\
\hline Biozeid 2.5\% 250 & 72.00 & 60.20 & 56.90 & 52.67 & $60.44 b$ \\
\hline Biozeid 2.5\% 200 & 72.00 & 60.00 & 56.80 & 51.50 & $60.08 \mathrm{c}$ \\
\hline Pritecto 9.4\% 300 & 72.00 & 63.48 & 60.50 & 52.83 & $62.42 a$ \\
\hline Pritecto 9.4\% 250 & 72.00 & 63.50 & 60.20 & 52.50 & $62.05 a$ \\
\hline Pritecto 9.4\% 200 & 72.00 & 63.20 & 60.00 & 52.00 & $61.80 \mathrm{~b}$ \\
\hline Bioarc 6\%300 & 72.00 & 69.40 & 64.38 & 57.38 & $65.92 a$ \\
\hline Bioarc 6\% 250 & 72.00 & 68.40 & 64.20 & 57.30 & $65.48 a$ \\
\hline Bioarc 6\% 200 & 72.00 & 68.20 & 64.00 & 57.20 & $65.35 a$ \\
\hline Control & 72.00 & 59.83 & 55.03 & 52.50 & $59.96 b$ \\
\hline \multirow[t]{2}{*}{ Means (A) } & $72.00 \mathrm{a}$ & $63.71 b$ & $59.90 c$ & $54.06 \mathrm{~d}$ & \\
\hline & \multicolumn{5}{|c|}{ Season 2014} \\
\hline Biozeid 2.5\% 300 & 70.25 & 66.58 & 61.90 & 56.90 & 63.91a \\
\hline Biozeid 2.5\% 250 & 70.25 & 66.40 & 61.50 & 55.90 & $63.51 b$ \\
\hline Biozeid 2.5\% 200 & 70.25 & 66.20 & 61.20 & 54.80 & $63.11 \mathrm{c}$ \\
\hline Pritecto 9.4\% 300 & 70.25 & 67.03 & 57.63 & 54.30 & 62.30a \\
\hline Pritecto $9.4 \% 250$ & 70.25 & 65.02 & 57.50 & 54.00 & $61.69 a$ \\
\hline Pritecto 9.4\% 200 & 70.25 & 65.00 & 57.30 & 53.90 & $61.61 b$ \\
\hline Bioarc 6\% 300 & 70.25 & 62.92 & 66.43 & 64.75 & $66.08 \mathrm{a}$ \\
\hline Bioarc 6\% 250 & 70.25 & 62.70 & 66.20 & 64.00 & $65.79 b$ \\
\hline Bioarc 6\% 200 & 70.25 & 62.50 & 66.00 & 63.98 & $65.68 \mathrm{c}$ \\
\hline Control & 70.25 & 60.83 & 57.38 & 53.43 & $60.47 b$ \\
\hline Means(A) & $70.25 a$ & $64.52 \mathrm{~b}$ & $61.40 \mathrm{c}$ & $57.59 \mathrm{~d}$ & \\
\hline
\end{tabular}

LSD $0.052013 \mathrm{~A}=8.50, \quad \mathrm{~B}=0.80 \quad$ LSD $0.05 \quad 2014 \quad \mathrm{~A}=5.80, \quad \mathrm{~B}=0.50$ 
Table 9. Effect of three bioinsecticides spray in preharvest to control peach fruit fly during postharvest on decay (\%) of guava fruits during 2013 and 2014 season

\begin{tabular}{llllll}
\hline Treatment g/100 I water & \multicolumn{5}{c}{ Storage period (week) } \\
\cline { 2 - 6 } & 1 & 2 & 3 & 4 & Means (B)
\end{tabular}

\begin{tabular}{|c|c|c|c|c|c|}
\hline \multirow[b]{2}{*}{ Biozeid 2.5\% 300} & \multicolumn{5}{|c|}{ Season 2013} \\
\hline & 6 & 7 & 8 & 8 & $7.25 a$ \\
\hline Biozeid 2.5\% 250 & 5 & 6 & 6 & 7 & $6.00 \mathrm{~b}$ \\
\hline Biozeid 2.5\% 200 & 5 & 6 & 7 & 8 & $6.50 \mathrm{c}$ \\
\hline Pritecto $9.4 \% 300$ & 8 & 9 & 9 & 10 & $9.00 \mathrm{a}$ \\
\hline Pritecto 9.4\% 250 & 5 & 9 & 10 & 10 & $8.50 \mathrm{~b}$ \\
\hline Pritecto 9.4\% 200 & 6 & 7 & 8 & 9 & $7.50 \mathrm{c}$ \\
\hline Bioarc 6\% 300 & 5 & 6 & 6 & 7 & $6 a$ \\
\hline Bioarc 6\% 250 & 4 & 5 & 5 & 6 & $5 b$ \\
\hline Bioarc 6\% 200 & 3 & 4 & 4 & 5 & $4 \mathrm{c}$ \\
\hline Control & 10 & 15 & 18 & 24 & $16.75 a$ \\
\hline \multirow[t]{2}{*}{ Means (A) } & $5.90 \mathrm{a}$ & 7.40 & $8.10 \mathrm{c}$ & $9.40 \mathrm{~d}$ & \\
\hline & \multicolumn{5}{|c|}{ Season 2014} \\
\hline Biozeid 2.5\% 300 & 7 & 8 & 9 & 10 & $8.50 \mathrm{a}$ \\
\hline Biozeid 2.5\% 250 & 6 & 7 & 7 & 8 & $7.00 \mathrm{~b}$ \\
\hline Biozeid 2.5\% 200 & 5 & 6 & 7 & 8 & $6.50 \mathrm{c}$ \\
\hline Pritecto 9.4\% 300 & 9 & 10 & 11 & 12 & $10.50 \mathrm{a}$ \\
\hline Pritecto $9.4 \% 250$ & 8 & 9 & 10 & 10 & $9.22 b$ \\
\hline Pritecto $9.4 \% 200$ & 8 & 10 & 11 & 12 & $10.25 c$ \\
\hline Bioarc 6\% 300 & 5 & 6 & 7 & 8 & $6.50 \mathrm{a}$ \\
\hline Bioarc 6\% 250 & 4 & 5 & 7 & 7 & $5.75 b$ \\
\hline Bioarc 6\% 200 & 2 & 4 & 5 & 6 & $4.25 c$ \\
\hline Control & 12 & 17 & 23 & 28 & $20 a$ \\
\hline Means (A) & $6.60 a$ & $8.20 b$ & $9.70 \mathrm{c}$ & $10.90 \mathrm{~d}$ & \\
\hline
\end{tabular}




\section{REFERENCES}

AOAC (2005). Official Methods of Analysis. $18^{\text {th }}$ Ed. Association of Official Analysis Chemists. Washington, DC, USA

Cheaour, F.C., J. Willemote, Y. Aruk, Y. Desjardin, P.M. Makhlouf and A. Gosselin (1990). A foliar application of calcium chloride delays postharvest ripening of strawberry. J. Ame. Sci., 115:789-792.

Chen, P.M. and W.M. Mellenthin (1981). Effects of harvest date on ripening capacity and post-harvest Life of d'Anjou pears. J. Ame. Soc. Hort. Sci., 106 (1): 38 - 42

David, B. and Whyte (2009). 1-MCP on banana. Food Sci., 12-16.

El-Seidy, R.M. (2000). Postharvest prestorage calcium treatments in relation to quality changes and storage ability of peaches. Ph.D. Thesis Alex. Univ., Alex., Egypt.

El-Saedy, R.M. and N.I. El-Naggar (2005). Effect of pre and postharvest calcium treatments on polyphon l oxidase and peroxidase activity of seedless guava fruits. Egypt. J. Appl. Sci., 20 (4): 224 - 231

El-Yaten, A. and A. Kader (1984). Controlled atmosphere alternatives to the postharvest use of sulfurs dioxide to inhibit the development of botrytis cinerea in table grapes, Univ. Calf., 3 : 160 - 164 .

Esam Eldin, B.M.K. and O.E. Nasr (2014). Effect of guava fruit color and size on fruit fly incidence in khartoum state.www. neppo. org/wp.content

Hanafy, A.H. (2000). Laboratory evaluation of some local food attraction of the med fly Ceratitis capitata (wied). Annal. Agric. Sc. Moshtohor, 38 (u):2517-2527.

Hanafy, A.H., A.A. Amed, A.M.Z. Mosallm and M.G.M. El-Sherief (2009).The efficiency of new natural compound as insecticides against peach fruit fly Bacterocero zomata (saunders). J. Agric. Mansoura Univ., 34 (1): 479-486.

Khyber, P. (2014). Insect pests and postharvest losses of guava (posted on bitlanders). www. bitlanders. com./biogs/insect
Lopez, A.C., A.C. Hernandez, F.G. Lara and O.P. Lopez (2003). Physio-chemical changes during ripening in storage of two varieties of prickly pear stored at $18^{\circ} \mathrm{C}$. J. Food Technol., 40 (5): 461 - 464.

Mahmoud, F.M. (2009). Susceptibility of the peach fruit fly Bacterocero zomata (saunders) (Diptera : Tephritidae) to three Entomopa thogenic Fungi. Egyptian J. Biol. Pest Control, 19 (2) : 169-179.

Michael, M. and A. Palmateer (2013). Florida Plant Disease Management Guide : Guava (psidium guajava). Publication, 232.

Muhammad, J.T., A.A. Nadeem and A.H. Ishfaq (2012). Effect of salicylic acid treatments on storage life of peach fruits cv. "Flordaking." J. Pak. J. Bot., 44 (1): 119 - 124.

Nurul, A. and H. Mosharraf (2012). Reduction of postharvest loss and prolonging the shelf. life of banana fruits through hot water treatment. J. Chem. Engin., IEB, $27: 1$.

Omayma, M.I., E.A.A. El-Moniem, A.S.E. Abd Allah and M.A.A. El-Naggar (2010). Influence of some postharvest treatments on guava fruits. Agric. and Biol. J. North Ame., 2151-2177.

Pen, J.E., R. Duncan, T. Vasquez and M. Hennessey (1999). Guava arthropod seasonality and control of fruit flies in South Florida. Proc. Fla. State Hort. Soc., 112-206-209.

Ponomarev, P.F. (1968). Changes in content of pectin and in activities of pectolytic enzymes during ripening and storage of pears. Tovaroredenie 3: 6 - 10 C.F. Fed. Sci. Technol. Abst., 2 (10): 1092.

Rahmann, G. and U. Akhsoy (2014). Control of fruit flies pest on guava fruit by using organic insecticide. Building organic bridges, johann Heinrich vonthunen-Institute, Braunschwei, Germany, Thuenen Rep., 675-678.

Rippon, L.E. and Trochoulias (2007). Ripening responses of banana to temperature, Aust. J. Exp. Agric., 16 (78): 140 - 144 SAS (1985).

Rosario, R.C., J.S. Guillen, E.B. Griffin and M.A.A.V. Lopez (2013). Irradiation effects on the chemical quality of guavas. Advance $\mathrm{J}$. Food Sci. and Technol., 5 (2): 90-98. 
Robert, E.P. and C.C. Ching (2014). Postharvest quality-maintenance guidelines, Fruit, nut and beverage crops, F-N-41.

SAS uses 'guide statistics for personal computers version $5^{\text {th }}$ Ed. SAS Inst. Cary $N$ Co.

Siddiqui, S., A. Brackmann, J. Sterif and Bangenrth (1996). Controlled atmosphere storage of Apples: cell wall compostion and fruit softening. J. Hort, Sci., 71 (4): 613-620 (C.F. Hort. Abst., 66 (11): 9219).

Singh, S.P. and R.K. Pal (2009). Ionizing radiation treatment to improve postharvest life and maintain quality of fresh guava fruit. Rad. Phys. and Chem., 78 (2): 135-140.
Snedecor, G.W. and W.G. Cochran (1980). Statistical Methods. $7^{\text {th }}$ Ed., $4^{\text {th }}$ printing, the Iowa state Univ. Press Ames., Iowa USA.

Steel, R.G.D and J.H. Torrie (1980). Principals and Procedures of Statistics. A biometrical approach. $2^{\text {nd }}$ MC Grow-Hill kogakusha Ltd., 633.

Yassin, N.M. and E.A. Tayel (2011). Regulating of ripening and maintaining the quality of banana fruits treated with 1-MCP. Exogenous Ethylene and storage temperature. Alex. Sci. Exchange J., $32: 3$.

Yavada, U.T. (1996). Guava (Psidium guajava L.). An exotic tree fruit with potential in south eastern United states .Hortsci.31:789794.

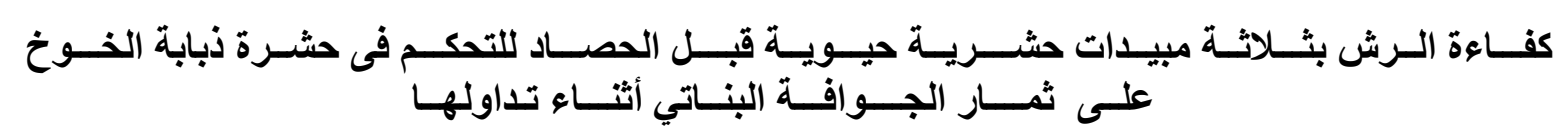

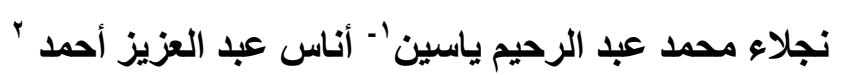

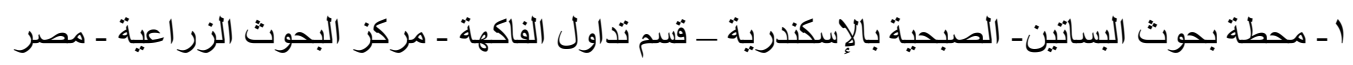

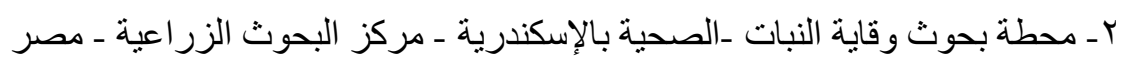

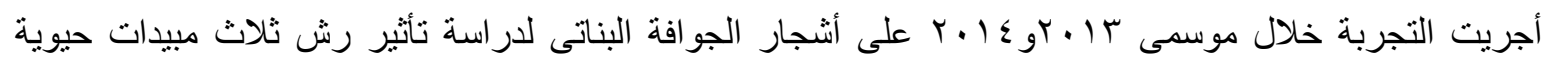

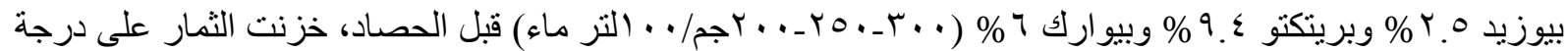

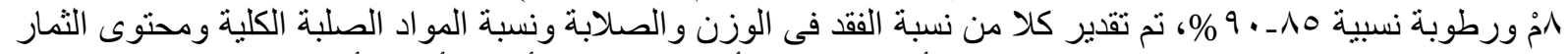

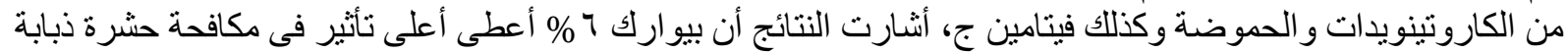

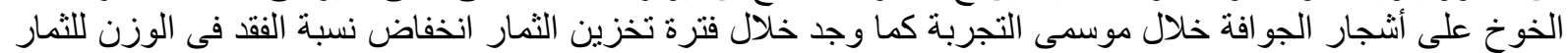

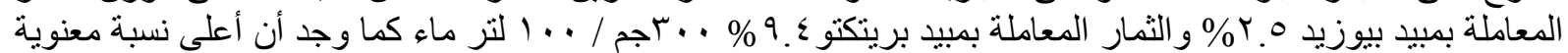

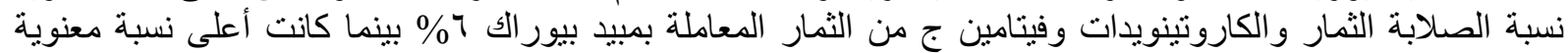

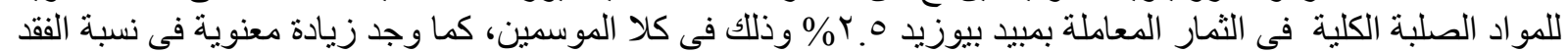

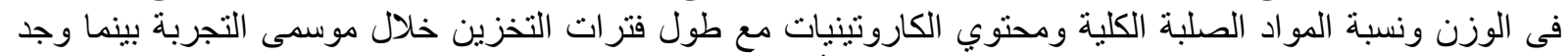
انخفاض معنوى فى نسبة الصلابة و الحموضة ونية وفيتامين ج أثناء التخزين. 